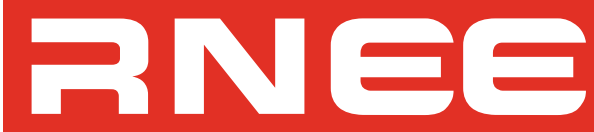

Revista Nicolaita de Estudios Económicos

Nueva Época

Instituto de Investigaciones Económicas y Empresariales
Universidad Michoacana de San Nicolás de Hidalgo

Volumen XVI, Número 1, Enero - Junio 2021, pp. 51 - 67

ISSN (Print): 1870 - 5464 ISSN (On-line): 2007 - 9877

DOI: 10.33110/rnee.v16i1.321

(Recibido: 13/octubre/2020; Aceptado: 01/marzo/2021)

\title{
Determinantes de la variación de precio semanal de cortes de carne vacunos en una región de Argentina
}

\section{Determinants of weekly prices of beef cuts in Argentina}

\author{
Mauro Finucci ${ }^{\text {a }}$ \\ Victoria Giordano ${ }^{b}$ \\ Ulises Glave ${ }^{c}$ \\ Fernando Delbianco d \\ Juan M.C. Larrosa ${ }^{\mathrm{e}}$ * \\ Gonzalo Ramírez Muñoz de Toro $\mathrm{f}$ \\ Juan I. Uriarte ${ }^{g}$
}

\section{Resumen}

Se estudia la evolución semanal de los precios de cortes de carne vacunos a través de datos en línea. Se modela dinámicamente las tasas de variación de precios de los cortes de carne como determinadas por las variaciones en precios de bienes sustitutos, insumos, diversas variables del mercado cambiario, shocks de oferta y efectos temporales. Se modela econométricamente y los resultados muestran efectos de sobreajuste de precios a corto plazo en diversos cortes, efecto de sustitución entre carnes alternativas de corto y largo plazo, y un rol limitado a cortes puntuales del tipo de cambio y efectos semanales.

Palabras claves: carne vacuna, fijación de precios, datos en línea, inflación.

Clasificación JEL: L11; Q11; C22

\begin{abstract}
We study the weekly evolution of prices of meat cuts using online data. We dynamically consider the rates of variation of prices of meat cuts as determined by variations in prices of substitute meats, inputs, various exchange market variables, supply shocks and temporary effects. We model econometrically and the results show effects of short-term price over-shooting in various cuts, substitution effect between short-term and long-term alternative meats, and a limited role of the exchange rate variables and weekly effects limited to specific cuts.

\footnotetext{
a, b, c Departamento de Economía, Universidad Nacional del Sur

d Departamento de Economía, Universidad Nacional del Sur, Instituto de Matemática de Bahía Blanca (IMABB)

* Departamento de Economía, Universidad Nacional del Sur, Instituto de Investigaciones Económicas y Sociales del Sur (IIESS)

Departamento de Derecho, Universidad Nacional del Sur, Hyperia

Hyperia

Autor de correspondencia jlarrosa@uns.edu.ar
} 
Keywords: beef, pricing, online data, inflation

JEL Codes: L11; Q11; C22

\section{Introducción}

La carne vacuna resulta un componente esencial en la dieta de muchos países. Específicamente, en Argentina debido a la ventaja comparativa de sus extensas llanuras consecuentes con grandes rebaños, este alimento resulta relativamente abundante y compone el 9,92\% del gasto de las familias (INDEC, 2013). Si bien los cambios culturales han afectado los patrones de consumo y la carne vacuna ha sido especialmente afectada, aún hoy el precio de la carne resulta una información sensible para determinar el acceso a la alimentación básica en el país sudamericano. Diversas investigaciones han aportado detalles respecto a la influencia de diversos determinantes respecto al precio. Siguiendo lo básico del análisis microeconómico los bienes sustitutos, complementarios, el ingreso, el precio de los insumos y diversos otros covariantes han jugado un papel en el traslado de sus efectos a los precios minoristas de cortes de carne.

Desde lo macroeconómico, como el tipo de cambio, al efecto de eventos naturales, como la sequía, puede generar impactos en el precio de los cortes de carne. ¿Cuánto de ello se puede percibir en la granularidad de la medición semanal de precios? En el caso del tipo de cambio, luego de un cambio de gobierno con alta inflación a finales de 2015 hasta mediados de 2016, se mantuvo en valores crecientes pero predecible dentro de los esquemas de política monetaria anunciados por el Banco Central de la República Argentina (BCRA). En mayo y agosto de 2018, sin embargo, inesperadas y fuertes devaluaciones se trasladaron a toda la canasta de productos y servicios de las familias. Del mismo modo y en base a los acuerdos de regulación del precio de combustibles, la subida del precio del dólar tiene un doble impacto en el precio de los combustibles. En el caso de la sequía de 2018, Argentina se encontraba hasta el 2017 en una fase ganadera cercana a la neutralidad, es decir ni en fase de liquidación (exceso de oferta) ni en fase de retención (escasez de oferta). Sin embargo, la sequía y su impacto en la producción forrajera llevaron a aumentos en la tasa de faena que puede afectar el ciclo ganadero y este comportamiento está generando impactos en el precio de la hacienda, insumo principal de los cortes.

Este trabajo aporta detalles sobre la influencia de diversos determinantes en la dinámica semanal de variación de precios de los cortes de carne provistos a grandes supermercados en una región de Argentina. Sin embargo, esta se encuentra en la región núcleo más productiva en términos de ganadería siendo, por ello, altamente influyente en la determinación del precio nacional promedio del producto. Por otro lado, no existe un antecedente de un estudio de este sector particular (corte de carne vacuno) a esta frecuencia (variación de precios semanales). Al trabajar con información obtenida mediante procedimientos de extracción automatizado de datos en línea (Uriarte y otros, 2019) se pudo analizar más de diez mil datos para las estimaciones. Al estilo de varias contribuciones las carnes han sido diferenciadas por diversos cortes los que representan más acabadamente la necesidad a cubrir en la alimentación familiar. Cada corte remite a una forma de cocción y presentación particular por ello tiene su propia demanda (Colella y Ortega, 2017). Asimismo, los oferentes del producto (carniceros) destacan diferencias de clientela en torno la materia grasa presente en el corte (Cáffaro Tommasiello y otros, 2021). Por esa razón investigaremos a cada corte como un producto individual con sus relaciones de sustitución respectivas.

El trabajo continua con la sección 2 donde se revisa la literatura, la sección 3 donde se exponen los datos a utilizar y la sección 4 se presenta los modelos econométricos, sus ajustes e interpretaciones de resultados. Finalmente, la sección 5 concluye el trabajo. 


\section{El mercado de los cortes de carne vacuna}

Durante la década de 2010 el mercado de carne vacuno en Argentina ha sufrido diversos shocks de política agropecuaria y externos que modificaron los precios. Hacia 2015 y 2016 el mercado recuperaba su perfil internacional con la liberación de diversos cortes para la exportación aunque el período 2015-2018 estuvo sujeto a diversos shocks de oferta de origen interno y externo (Eliçabe y otros, 2009). En otro estudio para Argentina (Pace Guerrero y otros, 2014) se demuestra que la carne tiene una elasticidad de gasto positiva y creciente en las familias así como una elasticidad precio negativa e inelástica.

Aunque ha pasado una década desde la crisis alimentaria, los mercados agrícolas mundiales siguen siendo susceptibles a un aumento en la volatilidad de los precios que puede ser causado por shocks de oferta y demanda. Las fuentes de crisis de la demanda incluyen crisis relacionadas con la salud animal (Van Asseldonk et al., 2000; Serra, 2011) y prohibiciones de importación por parte de los principales países importadores en respuesta a problemas de inocuidad alimentaria o de salud animal que causan un exceso de oferta en relación con la demanda (FAO, 2011).

Trabajos sobre determinación de precios en general identifican determinantes y aplican algún procedimiento econométrico de estimación. En el caso de Parcell y Schroeder (2007) emplea un modelo hedónico con datos mensuales para estimar el efecto de marcas en las ventas minoristas de carne vacuna y carne porcina. Los precios son modelados prestando atención a los descuentos y promociones. El trabajo muestra distintas características asociadas al precio de cortes de carne Premium, ubicaciones geográficas, tipos de negocio y estacionalidad. Assefa y otros (2017) modelan la volatilidad del precio de la carne de cerdo en Alemania. El trabajo estudia durante poco más de 10 años la transmisión de volatilidad de los costos de producción a los precios minoristas controlando por el poder de mercado de los productores. Los shocks de oferta y demanda hacen que los precios de los comestibles sean volátiles y, por lo tanto, impredecibles. El riesgo y la incertidumbre asociados con los precios volátiles a su vez aumentan la complejidad de las decisiones de gestión y de inversión (Piot-Lepetit, 2011; Rabobank, 2011). Assefa et al. (2017) mostró a través de entrevistas con 42 actores de la cadena alimentaria de la UE que la volatilidad de los precios es un desafío no solo para los agricultores sino también para otros actores de la cadena. Asimismo, para el mercado argentino Rossini y Depetris Guiguet (2008) estudia cómo los costos trasladan sus efectos a precios de manera asimétrica: es decir las alzas se transmiten con mayor intensidad que las bajas. En el mismo mercado, un trabajo de campo sobre carniceros revela que se observa una diferencia significativa en la frecuencia de compra de las medias reses mientras que todos poseen cámara fría (Cáffaro Tommasiello y otros, 2021). Destacan que los atributos de calidad de la carne vienen marcadas por el color como factor de frescura. La presencia de grasa intramuscular ayuda a distinguir también al tipo de cliente, optando algunos por cortes con grasa marmolada y otros con cortes magros (sin grasa visible).

Una segunda corriente de contribuciones trata el tema de la volatilidad de los precios, definida como la "parte" de los cambios de precios que no se pueden predecir por los fundamentales del mercado, los cuales en diferentes etapas de la cadena puede verse exacerbada por la posible transmisión de la volatilidad de los precios desde otras etapas de la cadena (Assefa et al., 2015). La transmisión de precios (de cambios de precios predecibles) es necesaria para la transmisión eficiente de las señales del mercado como base para decisiones económicas informadas (Chavas y Mehta, 2004). La transmisión de la volatilidad de los precios, por otro lado, implica la transmisión del riesgo y la incertidumbre y puede tener impactos negativos en la inversión en otras etapas de la cadena. Genchev y otros (2017) analizan el mercado búlgaro. Los precios en el sector de la carne en Bulgaria tienen las siguientes características: nivel no estimulante de los precios del ganado de carne engordado; 
la discrepancia entre la dinámica del precio al productor y los precios al por menor en los últimos años; establecimiento de "spread" relativamente grandes entre los precios al productor y los precios al por menor de la carne vacuna (ausencia de traslado de precios). Finalmente, Volpe et al. (2017) estudian los determinantes de precios en diferentes categorías de productos vendidos en supermercados. Las razones investigadas por el trabajo recaen en cambios en los precios de los insumos, cambios en la demanda, precios promocionales, controlando por la concentración de mercado y el tipo de establecimiento (comercio con fuertes promociones permanentes o con promociones temporarias). En el caso de la carne encuentran que la proporción de supermercados tradicionales como porcentaje de las firmas estudiadas está relacionada con la bajas de precios mientras que las comercios con promociones diarias están asociadas a precios más caros. Fakari et al. (2016) estudia la volatilidad en el mercado de carnes iraní. Utilizan datos semanales modelados como procesos GARCH y BEKK encontrando que la carne de cordero es más volátil que la de carne vacuna y aviar, siendo que la volatilidad de la primera está fuertemente relacionada con la segunda.

Una tercera corriente estudia el efecto del poder de mercado en el mercado de carnes. Tal es el caso de Weber y Anders (2007) que estudia el nivel de afectación del poder de mercado en los precios de carnicerías alemanas utilizando datos de cajero semanales. Calculan la rigidez de precios como un indicador de estabilidad en el precio medido por el número de semanas en los que se fijó precios en el corte de carne divido el número de semanas en los que se cambió el precio. El poder de mercado es también investigado en Hall y otros (1979) que presenta el caso de la influencia de la concentración en el spread del precio de la carne a nivel de productor y la carne en el mostrador (McFall Lamm (1981) representa también un trabajo inicial al respecto). Encuentran que la concentración está asociada positivamente con el nivel de spread. Wohlgenant y Mullen (1987) también modelan la brecha entre el precio de granja y el precio minorista con una especificación basada en los márgenes de rendimiento para el precio de la carne en Estados Unidos, utilizando datos anuales.

Una cuarta corriente de literatura realiza estudios de demanda de cortes de carne propiamente dichos explican cómo las ventas han sido afectadas por determinantes específicos. Por ejemplo, Lusk y Norwood (2055) enfoca el problema de la heterogeneidad de los cortes de carnes como productos en sí mismos con demandas propias. Efectos directos y cruzados en el precio son modelados tendientes a identificar la prima de precio por los cortes de carne. Es un elemento indispensable para identificar la demanda de carne modernamente. En el caso de Charlebois y otros (2016) observan que los patrones de consumo han cambiado mucho en los últimos años en todo Occidente (Sahin y otros, 2014) observándose aumentos de precios a lo largo de todo Occidente. En las naciones desarrolladas el consumo de carne se siente afectado por temas éticos y sanitarios relativo a su consumo (Beardsworth and Bryman, 2004). En un trabajo de campo realizado en Canadá obtienen información que confirma que pese al aumento sostenido del precio de la carne el consumo se mantiene debido a motivaciones alimentarias. Capps y Nayga (1991), por su parte, analizan datos de cajeros de supermercados (scanner data) en frecuencia semanal que les permite distinguir el efecto de promociones sobre las ventas de diversos cortes de carne, entre ellos: brisket, chuck, ground, loin, rib y round. Luego emplea un modelo de regresión lineal para asociar el precio de cada corte a diversas carnes sustituto y complementario, insumos, estacionalidad y diversos dummies temporales.

En definitiva, ¿qué determinantes afectan a la fijación de precios de la carne al mostrador en Argentina? Los costos de producción naturalmente transmiten variabilidad al precio respectivo de cada corte de carne junto con atributos propios de cada corte. Es decir, cada corte está asociado a diferentes preparaciones y, si bien puede haber sustituciones, no lo es para todos los casos: los cortes de carne tradicionalmente asados no se usan para guisados, por ejemplo. Por lo tanto, cada corte puede trabajarse individualmente y ese será el enfoque del presente trabajo. 


\section{Datos y variables}

Para analizar el impacto de los determinantes en la evolución de los precios de los cortes de carne vacuna agrupamos a los mismos en costos, precios de bienes relacionados y shocks de oferta y demanda. Los datos fueron descargados de la red mediante el procedimiento de raspado de web (webscraping) y pertenecen al proyecto IPC Online (Uriarte y otros, 2019). Todos los datos son en frecuencia semanal, desde la primera semana de Diciembre de 2015 hasta la cuarta semana de Diciembre de 2018 (148 semanas). Cada mes es dividido en 4 semanas cada uno (del 1 al 7 corresponde a 1ra semana, del 8 al 14 corresponde a 2da semana, del 15 al 21 corresponde a la 3ra semana, y del 22 al 28 corresponde a la 4 ta semana de cada mes). A este nivel de frecuencia si las variables resultan significativas determinarían un grado alto de sensibilidad al impacto de las variables explicativas. Es un período muy corto de tiempo y se consideran habitualmente datos de alta frecuencia.

Para evaluar el efecto de diversos determinantes a la dinámica semanal de precios proponemos un modelo econométrico que tome en cuenta variables relevantes medidas en frecuencia semanal.

$$
P_{i t}=f\left(P R_{t}, I_{t}, S O_{t}, E X_{t}, E T_{t}\right)
$$

Es decir, las variables dependientes $P_{i t}$ son las variaciones semanales de diversos cortes de carne vacuna dependen de las variaciones de precios de precios de cortes de carne relacionados $P R_{t}$ evolución del precio de insumos $I_{t}$, shocks de oferta $S O_{t}$ representados por los valores de exportaciones, variables que representan al mercado cambiario $E X_{t}$ y finalmente $E T_{t}$ que representa diversas variables de efecto temporal tanto semanal, mensual y anual para capturar efectos estacionales.

La necesidad de distinguir los cortes de carne está basada en que la demanda distingue distintos usos alimentarios a cada corte y por lo tanto, son productos específicos con demandas específicas. En el caso de Argentina, Colella and Ortega (2017) presentan el resultado de experimento en base a encuestas realizados en carnicerías de Buenos Aires. Relevan datos de encuestas en carnicerías y supermercados buscando determinar si los clientes distinguen aspectos de calidad en los cortes de carne asociados al precio. Dos tipos de canales de comercialización distinguen el mercado argentino: supermercados y carnicerías tradicionales. En el caso de las carnicerías los consumidores apelan al consejo del carnicero para distinguir cortes de mejor calidad, aun cuando los supermercados puedan ofrecer mejores precios. Del mismo modo, las carnes de distinto origen en algún punto pueden competir entre ellas en este mercado, es decir puede existir efecto sustitución entre ellas (Pace Guerrero y otros, 2014) debido a variaciones en los precios relativos, patrones culinarios específicos, fiestas asociadas con ciertos alimentos o cambios en los gustos de los consumidores. Es por ello que también se considera en nuestro análisis el efecto del precio de carne de pescado, ave (pollo) y cerdo en la variación de precio de los cortes de carne vacuna.

En nuestro trabajo obtenemos datos de la segunda primera fuente de datos y a lo largo del trabajo se analizó la variación semanal de precios de los siguientes grupos de productos Carnes y Cortes delanteros y traseros de carne vacuna fresca y de los siguientes cortes específicos: Asado, Bife ancho, Carnaza común, Falda, Hueso con carne, Paleta, Vacío, Aguja, Bola de lomo, Cuadrada, Nalga y Peceto. Las variables a estimar son las variaciones de precios semanales de los siguientes cortes y agrupamientos (nombre y código): 
Variable $P_{i t}$

- Carnes v112: Agrupamiento de todas las carnes rojas y blancas

- Carne vacuna v1121: Agrupamiento de todos los cortes de carnes vacunas

- Cortes delanteros y traseros de carne vacuna fresca v11211: Agrupamiento de cortes delanteros

- Asado v1121101

- Bife ancho v1121102

- Carnaza común v1121103

- Falda v1121105

- Hueso con carne v1121106

- Paleta v1121108

- Vacío v1121110

- Aguja v1121113

- Bola de lomo v1121122

- Cuadrada v1121123

- Cuadril v1121124

- Nalga v1121126

- Peceto v1121127

\section{Variable $P R_{t}$}

Las variaciones de precios de bienes relacionados en términos de consumo a ser contrastadas como bienes sustitutos comprenden:

- Otras carnes rojas frescas v1122

- Carne de ave v1123

- Aves frescas y congeladas v11231

- Pescados frescos y congelados v1124

\section{Variables $I_{t}$}

Las variables determinantes variación de precios incluidas en el análisis como precios de los insumos. En nuestro caso tomos los datos del precio del kilo vivo de novillito en pie en el Mercado de Hacienda de Liniers (http://www.mercadodeliniers.com.ar/) en datos semanales.

- Precio insumo Novillito: Variación del precio de novillito por kilo vivo en el mercado de Liniers

- Precio minorista de Combustibles

Los shocks de oferta interno serán representados por las exportaciones de carne vacuna. Para ello se ha tomado información del Instituto de Promoción de la Carne Vacuna Argentina (IPCVA) disponible en su sitio (http://ww.ipcva.com.ar). Aquí los datos fueron transformados dado que su frecuencia original es mensual. Se tomó la variación mensual y se la prorrateó acumuladamente en semanas de modo que la variación acumulada semanal totaliza la variación mensual registrada. 


\section{Variables $\mathrm{SO}_{t}$}

Las variables representan el destino sustituto del mercado interno de la producción ganadera argentina representada por variaciones de las exportaciones de carne en diferentes representaciones. Los datos originales se presentan en frecuencia mensual pero fueron transformadas a frecuencia semanal mediante el prorrateo de la tasa de variación mensual en cuatro periodos semanales que acumulan la variación mensual observada. Entonces las variables creadas fueron:

- Exportaciones totales en dólares: ExpUSD

- Exportaciones totales en kilogramos: EXPKg

- Exportaciones totales en kilos por dólar: EXPUSD-Kg

\section{Variables $E X_{t}$}

Otras variables de control serán del mercado cambiario y diversas métricas de las series de cotización del dólar comprador y vendedor de la casa Pullman de Bahía Blanca;

- Precio de compra del dólar: compra

- Precio de venta del dólar: venta

- Dólar promedio venta-compra: dolarprom

- Brecha = venta - compra: brecha del dólar (como indicador de inestabilidad cambiaria).

- Porcentaje = venta - compra / Promedio porcentaje (como indicador de inestabilidad cambiaria)

- Variación precio compra: varcpa

- Variación precio venta: varvta

- Desvío estandar intrasemanal precio compra: como indicador de volatilidad cambiaria

- Desvío estandar intrasemanal precio venta: como indicador de volatilidad cambiaria

- Cantidad de operaciones por dia de operación semanal: cantoperac; como indicador de inestabilidad/incertidumbre cambiaria

- Curtosis del precio de compra: kurtc

- Curtosis del precio de venta: kurtv

- Coeficiente de asimetría del precio de compra: skewpc

- Coeficiente de asimetría del precio de venta: skewpv

\section{Variables $E T_{t}$}

Luego se agregaron variables binarias para capturar el efecto temporal.

- Dummy semanal: Binaria para semana 1, 2 y 3 de cada mes. Semana de referencia: semana 4

- Dummy mensual: Mes de referencia es mes 11. Luego se crean 11 dummies para representar cada mes excepto el mes de referencia

- Dummy anual: Año de referencia 2016. Binaria en cada año distinto del año de referencia. 
Definidas las variables la siguiente sección describe el método econométrico aplicado junto con la interpretación de los resultados.

\section{Análisis de resultados ${ }^{1}$}

Estimamos mediante un modelo de rezagos distribuidos, mediante el comando ARDL, disponible en el software Stata, de Kripfganz y Schneider (2018), que automatiza mediante los criterios de información la cantidad de rezagos de las variables en el ajuste de corto plazo (las diferencias) y de largo plazo (los niveles). No solo resulta adecuado por la posible relación de largo plazo y ajuste entre los precios de los distintos cortes de carne, sino que posibilita el análisis de muchas regresiones en simultáneo, característica deseable dada la base de datos que se trabaja.

Como contraste utilizamos MCO ordinarios con cuatro rezagos para contabilizar por las posibles interdependencias semanales, a la vez que incluimos dummies para controlar no solo la semanalidad sino estacionalidad de mayor frecuencia temporal entre meses y años. Empezaremos describiendo estos resultados..

El análisis de los resultados de las regresiones de mínimo cuadrados ordinarios muestran que las carnes como grupo de productos mantienen una correlación negativa muy significativa con pescado fresco, es decir que subas en la variación de precios de carnes se asocian con bajas en a variación del segundo. Este resultado se enmarca en el rol de sustitutos de ambos. Extrañamente ese rol no se observa en otros cortes alternativos como carne de cerdo o ave. Las exportaciones, expresada en términos de $\mathrm{kg} / \mathrm{dó}$ lar exportado, afectan también negativamente la tasa de variación de precios del grupo de carnes. El año 2017, previo a la sequía de 2018, marcó también un período de menores variaciones de precios mientras que el mes de septiembre se destaca estacionalmente como un mes de aceleración de las variaciones promedios superiores al resto del período de estudio.

El subgrupo de Cortes delanteros y traseros de carne vacuna fresca mantiene los mismos determinantes que el grupo Carnes exceptuando la estacionalidad de septiembre aunque el nivel también afecta positivamente las variaciones de precios, es decir a mayores precios las variaciones han sido más significativas.

Dentro de los cortes específicos,

- Asado muestra también un efecto de sustituto con respecto al pescado fresco, efecto negativo en las variaciones al año 2017, estacionalidad positiva en mayo y junio, efecto arrastre atenuados en primero y segundo orden.

- Bife Ancho ha sido el que ha mostrado más efectos detectados. Se mueve positivamente acompañando el nivel general de grupo de Carnes, señalando marcadas bajas mensuales desde enero hasta septiembre comparado con el mes de referencia (diciembre), sin efectos de arrastre atenuados.

- Carnaza común muestra, por su parte, movimientos de ajuste positivo a la variación del insumo (novillito), un shock negativo particular en el año 2016, estacionalidad en el mes de junio y efecto arrastre atenuado.

- Falda, también presenta un shock negativo en 2016 y estacionalidad negativa aunque en enero y febrero, con efecto arrastre atenuado.

\footnotetext{
${ }^{1}$ Las tablas de ambas estimaciones puede descargarse desde el siguiente enlace:

https://drive.google.com/open?id=1tbZcmIe7pzuEAGRGK5iANVCWpuS5j67N
} 
- Hueso con carne solo muestra una reacción positiva al indicador de inestabilidad cambiaria.

- Paleta, a su vez, marca estacionalidad negativa en junio con efecto arrastre menguado.

- Vacío muestra variación paralela al del grupo de Carnes, estacionalidad positiva en julio y negativa en octubre y efectos arrastres suavizados.

- Aguja solo presenta estacionalidad positiva en octubre.

- Bola de lomo muestra efecto de alzas en la segunda semana y con la inestabilidad cambiaria, estacionalidad negativa con enero y mayo y efecto arrastre menguado.

- Cuadrada muestra estacionalidad negativa en junio junto a efecto arrastre suavizado.

- Nalga muestra variación paralela al del grupo de Carnes y negativa con pescado fresco, estacionalidad positiva en octubre y efectos arrastres suavizados.

- Finalmente, Peceto sólo registra efectos temporales con semanalidad positiva en la primera semana y efectos negativos en la aceleración de precios en 2016 y 2017 en presencia de efecto arrastre 
Tabla 1

Resumen de los resultados econométricos de $\mathrm{MCO}$

\begin{tabular}{|c|c|c|c|c|c|}
\hline Variables & Carnes relacionadas & Insumos & Shock de oferta & Mercado cambiario & Oferta temporal \\
\hline Carne vacuna & $\begin{array}{c}\text { Pescado fresco } \\
(-)\end{array}$ & & $\begin{array}{c}\text { Exportaciones } \\
\mathrm{kg} / \mathrm{USD}(-)\end{array}$ & & $\begin{array}{c}\text { Año } 2017\left({ }^{(-)}\right. \\
\text {septiembre (+) }\end{array}$ \\
\hline $\begin{array}{l}\text { Cortes } \\
\text { delanteros y } \\
\text { traseros de carne } \\
\text { vacuna fresca }\end{array}$ & $\begin{array}{c}\text { Pescado fresco } \\
(-)\end{array}$ & & $\begin{array}{l}\text { Exportaciones } \\
\mathrm{kg} / \text { USD (-) }\end{array}$ & & Año 2017 (-) \\
\hline Asado & $\begin{array}{c}\text { Pescado fresco } \\
(-)\end{array}$ & & & & $\begin{array}{c}\text { Año } 2017(-) \\
\text { mayo, junio } \\
\quad(+) \\
\text { Efecto arrastre }\end{array}$ \\
\hline Bife ancho & & & & & $\begin{array}{c}\text { Enero a } \\
\text { septiembre (-) }\end{array}$ \\
\hline $\begin{array}{l}\text { Carnaza } \\
\text { común }\end{array}$ & & Novillito $(+)$ & & & $\begin{array}{c}\text { Año } 2016(-) \\
\text { junio (-) } \\
\text { Efecto arrastre }\end{array}$ \\
\hline Falda & & & & & $\begin{array}{c}\text { Año } 2016(-) \\
\text { enero, febrero } \\
(-) \\
\text { Efecto arrastre }\end{array}$ \\
\hline $\begin{array}{l}\text { Hueso } \\
\text { con carne }\end{array}$ & & & & $\begin{array}{l}\text { Cantidad de } \\
\text { operaciones } \\
\text { semanales }(+)\end{array}$ & \\
\hline Paleta & & & & & $\begin{array}{c}\text { Junio (-) } \\
\text { Efecto arrastre }\end{array}$ \\
\hline Vacío & Carnes $(+)$ & & & & $\begin{array}{c}\text { Julio }(+) \\
\text { octubre }(-) \\
\text { Efecto arrastre }\end{array}$ \\
\hline Aguja & & & & & Octubre $(+)$ \\
\hline $\begin{array}{l}\text { Bola de } \\
\text { lomo }\end{array}$ & & & & $\begin{array}{l}\text { Cantidad de } \\
\text { operaciones } \\
\text { semanales }(+)\end{array}$ & 2 da semana $(+)$ \\
\hline Cuadrada & & & & & $\begin{array}{c}\text { Junio (-) } \\
\text { Efecto arrastre }\end{array}$ \\
\hline Nalga & $\begin{array}{c}\text { Carnes }(+) \\
\text { Pescado fresco }(-)\end{array}$ & & & & $\begin{array}{c}\text { Octubre }(+) \\
\text { Efecto arrastre }\end{array}$ \\
\hline Peceto & & & & & $\begin{array}{c}\text { 1ra semana (+) } \\
\text { Año } 2016(-) \\
\text { Año } 2017(-) \\
\text { Efecto arrastre }\end{array}$ \\
\hline
\end{tabular}

Fuente: Los autores. Las tablas completas se exhiben en: https://drive.google.com/open?id=1tbZcmIe7pzuEAGRGK5iANVCWpuS5j67N 
Los resultados del modelo ADRL se exponen a continuación. En el caso del agrupamiento Carnes los resultados reflejan que efectos son similares en el corto (rezagos) como en el largo plazo (diferencias). Hay un efecto positivo del capítulo de carnes en general (del cual el corte es una parte), y un efecto negativo de otras carnes que son sustitutos, como otras carnes rojas, pescado o pollo en el corto plazo. De los efectos de sustitución, el del pollo tiene un efecto marginal en el precio mucho más grande. En el efecto de ajuste, es de esperar lo que se observa en la significatividad de la carne vacuna. En otras palabras, existe casi un efecto de ajuste muy rápido del corte con respecto al resto de los cortes de carne vacuna. Por otro lado, no hay efectos de significatividad de insumos, exportaciones o efectos de las dummies estacionales. Finalmente, por criterio de información, la cantidad de rezagos en el corto plazo quedo reducido a sólo uno.

En el caso de los cortes delanteros y traseros también se observan similitudes entre el corto y largo plazo respecto al efecto positivo del capítulo de carnes en general, y al efecto negativo del pollo como sustituto. Por otra parte, otros sustitutos (otras carnes rojas frescas y pescado) resultan significativos solamente en el corto plazo. También se debe considerar el efecto positivo del precio de kilo vivo de novillito (insumo). Respecto al efecto de ajuste se visualiza que el mismo es muy rápido, siendo este efecto significativamente mayor que el de la media general de todos los cortes.

En cuanto al asado, se observa nuevamente la existencia de un efecto positivo del capítulo carnes en general, y un efecto negativo de los sustitutos (pollo y pescado) tanto en corto como en largo plazo. Respecto a los efectos de sustitución, el pescado cuenta con un efecto marginal en el precio notoriamente superior. Si se considera el corto plazo, se encuentra además un efecto positivo por parte del insumo. En cambio, en el largo plazo esta última variable no es significativa, y se incluye el efecto negativo de las exportaciones. Por último, puede observarse un efecto de ajuste muy veloz, aún mayor que el observado en cortes delanteros y traseros.

Analizando el bife ancho, se evidencia que en el corto plazo sólo resulta significativa la variación del precio de compra del dólar (inestabilidad cambiaria) con efecto negativo. En cambio, en el largo plazo no sólo resulta significativa esta variable sino también el efecto negativo del pescado, teniendo éste un considerable efecto marginal en el precio del corte. Por otra parte, el efecto de ajuste de esta variable es el más veloz de todos los cortes analizados.

Respecto a la carnaza común, se visualiza que en el corto plazo únicamente resulta significativa la variable de pescados, manifestando un efecto marginal relativamente bajo. En tanto, en el largo plazo la única variable significativa es la del pollo. Por último, la velocidad de ajuste observada para esta variable es una de las más bajas entre todos los cortes analizados.

Examinando la falda, se destaca que tanto en el corto como en el largo plazo no hay significatividad de los bienes sustitutos a excepción del pescado en el corto plazo, cuyo efecto marginal es igualmente bajo. Además, las exportaciones resultan significativas para ambos horizontes temporales, presentando un efecto positivo en el corto plazo mientras que en el largo plazo es negativo. Por otro lado, la inestabilidad cambiaria resulta significativa para el largo plazo. Para finalizar con esta serie, se observa que su velocidad de ajuste es cercana a la media del resto de los cortes.

Continuando el análisis de las series, se observa que en el hueso con carne la única variable que resulta significativa, tanto en el largo como en el corto plazo, es el capítulo de carnes en general. A su vez, la velocidad de ajuste que se manifiesta es similar a la observada en la falda.

$\mathrm{Al}$ estudiar la serie correspondiente a la paleta, se destaca que otras carnes rojas frescas (cerdo) es el único sustituto que resulta significativo manifestando un efecto positivo, y únicamente en el largo plazo. Además, se evidencia un comportamiento similar para las exportaciones en el corto plazo. Por último, al observar la velocidad de ajuste de esta serie se puede considerar que presenta un valor alto. 
Resulta llamativo que al analizar la serie del vacío no se observan resultados significativos en las variables correspondientes a los bienes sustitutos tanto en el corto como en el largo plazo. Sin embargo, puede observarse un efecto positivo respecto al insumo novillo en el corto plazo significativo. A su vez, la velocidad de ajuste de esta serie es alta.

Con respecto a la aguja se visualiza que en el corto plazo el capítulo de las carnes en general y el pollo resultan significativos. A su vez, en el largo plazo se observa que el pescado y las exportaciones resultan significativos, destacándose el signo positivo de la primera. Por último, la velocidad de ajuste se aproxima a la media.

Analizando el bife de lomo se destaca que las variables correspondientes para la semana uno y la semana dos resultan significativas y positivas para el corto plazo, como también lo es el cerdo. En cambio, el pescado que también es significativo, muestra un signo negativo. Estos resultados del cerdo y el pescado son similares para el largo plazo. Al mismo tiempo, la velocidad de ajuste es levemente inferior al promedio.

La serie de la cuadrada presenta al pescado, exportaciones, e inestabilidad del tipo de cambio como significativas para el largo plazo, resultado que se repite en el corto plazo agregándose la variable de semana uno. Resulta notorio el signo positivo y el elevado efecto marginal de la variable pescado en el corto plazo, que luego se revierte a negativo en el largo plazo, pero manteniendo un alto efecto marginal. También es muy elevada la velocidad de ajuste de esta serie.

El cuadril muestra resultados distintivos ya que no presenta ninguna variable significativa tanto en el corto como en el largo plazo. A su vez, su velocidad de ajuste es levemente superior a la media.

Respecto a la nalga, las únicas variables significativas en el corto plazo son las de la primera y la tercera semana, ambas de signo negativo. En el largo plazo se muestra un efecto positivo del capítulo de carnes en general, acompañado de un efecto negativo del sustituto otras carnes rojas frescas. Por otra parte, la velocidad de ajuste de este corte resulta de las más rápidas del análisis.

Al observar el peceto, puede notarse el efecto positivo de la variación del precio del dólar tanto en el corto como en el largo plazo, enfatizándose dicho efecto en este último. En el corto plazo resultan además significativos los efectos negativos del cerdo y del pollo como sustitutos, como también se presenta un efecto positivo en la segunda semana. Por otra parte, en el largo plazo pueden observarse también un efecto negativo por las exportaciones y un efecto positivo respecto al novillo. La velocidad de ajuste de este corte resulta baja respecto a la media. 
Tabla 2

Resumen de los resultados econométricos de ARDL

\begin{tabular}{|c|c|c|c|}
\hline Variables & Corto plazo & Largo plazo & Ajuste \\
\hline Carne vacuna & $\begin{array}{l}\text { Carnes }(+) \\
\text { Otras carnes rojas }(-) \\
\text { Carne de ave }(-) \\
\text { Pescados frescos }(-)\end{array}$ & $\begin{array}{l}\text { Carnes }(+) \\
\text { Otras carnes rojas }(-) \\
\text { Carne de ave }(-) \\
\text { Pescados frescos }(-)\end{array}$ & $\begin{array}{l}- \\
0.987 * * \\
*\end{array}$ \\
\hline $\begin{array}{l}\text { Cortes } \\
\text { delanteros y } \\
\text { traseros de carne } \\
\text { vacuna fresca }\end{array}$ & $\begin{array}{l}\text { Carnes }(+) \\
\text { Otras carnes rojas }(-) \\
\text { Carne de ave }(-) \\
\text { Insumo }(+)\end{array}$ & $\begin{array}{l}\text { Carnes }(+) \\
\text { Carne de ave (-) }\end{array}$ & $\begin{array}{l}- \\
1.480 * * \\
*\end{array}$ \\
\hline Asado & $\begin{array}{l}\text { Carnes }(+) \\
\text { Otras carnes rojas }(-) \\
\text { Carne de ave }(-) \\
\text { Insumo }(+)\end{array}$ & $\begin{array}{l}\text { Carnes }(+) \\
\text { Otras carnes rojas }(-) \\
\text { Carne de ave }(-) \\
\text { Pescados frescos }(+) \\
\text { Exportaciones }(-)\end{array}$ & $\begin{array}{l}- \\
1.678^{* *} \\
*\end{array}$ \\
\hline Bife ancho & Variación precio compra (-) & $\begin{array}{l}\text { Pescados frescos (-) } \\
\text { Variación precio compra (-) }\end{array}$ & $\begin{array}{l}- \\
1.967 * * \\
*\end{array}$ \\
\hline Carnaza común & Pescados frescos (-) & Carne de ave (-) & $\begin{array}{l}- \\
0.604 * * \\
*\end{array}$ \\
\hline Falda & $\begin{array}{l}\text { Pescados frescos }(-) \\
\text { Exportaciones }(+) \\
\text { Variación precio compra }(+) \\
\text { Falda }(+) \\
\text { Carnes }(+) \\
\text { Carne de ave }(+)\end{array}$ & Variación precio compra (-) & $\begin{array}{l}- \\
1.005 * *\end{array}$ \\
\hline $\begin{array}{l}\text { Hueso } \\
\text { con carne }\end{array}$ & $\begin{array}{l}\text { Carnes }(+) \\
\text { Otras carnes rojas }(+) \\
\text { Carne de ave }(+) \\
\text { Carnes }(+) \\
\text { Otras carnes rojas }(+)\end{array}$ & & $\begin{array}{l}- \\
0.961 * * \\
*\end{array}$ \\
\hline Paleta & $\begin{array}{l}\text { Exportaciones } \mathrm{Kg}(+) \\
\text { Otras carnes rojas }(-) \\
\text { Variación precio compra }(+) \\
\text { Pescados frescos }(-) \\
\text { Carnes }(-) \\
\text { Carne de ave }(+) \\
\text { Otras carnes rojas }(-) \\
\text { Paleta }(+)\end{array}$ & Otras carnes rojas $(+)$ & $\begin{array}{l}- \\
1.557^{* *} \\
*\end{array}$ \\
\hline Vacío & $\begin{array}{l}\text { Insumo }(+) \\
\text { semana } 2(+) \\
\text { Otras carnes rojas frescas }(+) \\
\text { Carnes }(+) \\
\text { Vacío }(+)\end{array}$ & & $\begin{array}{l}- \\
1.340 * * \\
*\end{array}$ \\
\hline
\end{tabular}


Aguja

Bola de

lomo

Cuadrada

Cuadril

Nalga

Peceto
Carnes $\left(1^{\circ}\right.$ y $3^{\circ}$ rezago $)(+)$

Carne de ave $\left(1^{\circ}\right.$ a $3^{\circ}$ rezagos) (-)

Exportaciones $(+)$

Carne de ave $\left(2^{\circ}\right.$ y $3^{\circ}$ rezago $)(+)$

Otras carnes rojas

(diferencias y $1^{\circ}$ y $2^{\circ}$

rezago) $(+)$

Pescados frescos $(-)$

semana $1(+)$

semana $2(+)$

Carnes $(+)$

Pescados frescos

(diferencias y $1^{\circ}$ y $3^{\circ}$

rezago) $(+)$

Exportaciones $\mathrm{Kg}$

(diferencias $(+)$ y $2^{\circ}$

rezago $(-))$

Variación precio compra (-)

semana $1(+)$

Otras carnes rojas $(+)$

Cuadrada $(+)$
Pescados frescos (-)

Exportaciones $(+)$

Otras carnes rojas $(+)$

Pescados frescos (-)
$0.994 * *$

$0.842 * *$
Pescados frescos

Exportaciones

Variación precio compra
$1.526^{* *}$

$*$ semana $1(-)$

Exportaciones $\mathrm{Kg}(-)$

Nalga (+)

Otras carnes rojas

(diferencias $(-), 1^{\circ}$ y $2^{\circ}$

rezagos) $(+)$

Carne de ave (-)

Variación precio compra $(+)$

semana $2(+)$

Pescados frescos $(+)$

Peceto $\left(2^{\circ}(+)\right.$ y $3^{\circ}(-)$ rezago $)$
Carnes (+)

Otras carnes rojas (-)

Exportaciones $\mathrm{Kg}(-)$

Insumo $(+)$

Variación precio compra $(+)$
$1.188^{* *}$

$1.795 * *$

$*$

$0.506^{* *}$

*

Fuente: Los autores. Las tablas con resultados se exponen en:

https://drive.google.com/open?id=1tbZcmIe7pzuEAGRGK5iANVCWpuS5j67N 


\section{Conclusiones}

En esta contribución se busca dar luz a qué efectos han tenido diversos determinantes teóricos sobre la evolución del precio de cortes de carne provistos en supermercados en Argentina. Particularmente el trabajo explica la evolución en frecuencia semanal lo que permite determinar una dinámica no provista por las estadísticas oficiales. En este sentido, se recalca que los cortes están correlacionados por diversos determinantes en diversa medida dado que existe heterogeneidad de resultados. En gran parte de los cortes, al acelerarse la inflación semanal de los mismos, se verifica una desaceleración de cortes sustitutos, como pescado, pollo y cerdo con diferente efecto de rezagos en el tiempo y en algunos casos con efectos opuestos a los esperados. Se detectaron diferentes momentos de estacionalidad aunque el efecto semanal fue detectado en muy pocos cortes (vacío, bola de lomo, cuadrada, peceto).

Del mismo, las formas econométricas adoptadas detectaron en pocos casos (falda, paleta, cuadrada) un efecto de tipo de cambio en la aceleración de los precios de los cortes. Luego, entre el corto y largo plazo el ajuste se produce muy fuertemente en general, con alta probabilidad de sobreajuste excepto en dos casos (carnaza común y peceto).

Las exportaciones juegan un rol afectando la aceleración de precios en los cortes agrupados (cortes de carne vacuna y carnes) siendo más diverso su efecto cuando se estudian los cortes particulares y dimensiones temporales: a corto plazo, las exportaciones aceleran la falda y paleta y desaceleran nalga, mientras que en el caso de asado, nalga, peceto la desacelera y en el caso de aguja la acelera en el largo plazo.

La aceleración/desaceleración dentro del contexto de alta inflación con Argentina permite distinguir una dinámica poco estudiada debido básicamente a la dificultad de tomar los datos. El presente trabajo aprovecha para eso la posibilidad de obtener grandes de masas en línea.

Como futuras líneas de investigación para este trabajo queda por explorar el rol de sustitutos entre los propios de carne así como potencialmente adoptar otras formas econométricas que puedan capturar mejor potenciales efectos semanales (de comercialización) así como considerar el efecto de otros insumos de origen animal, no sólo el novillito que hemos tomado como insumo principal.

\section{Financiación}

Este trabajo ha sido financiado por el Proyecto de Grupo de Investigación 24/E159 de la Universidad Nacional del Sur. 


\section{Referencias}

Assefa, T.T., M.P.M. Meuwissen, C. Gardebroek and A.G.J.M. Oude Lansink (2017), "Price and Volatility Transmission and Market Power in the German Fresh Pork Supply Chain” Journal of Agricultural Economics 68(3): 861-880; doi: 10.1111/1477-9552.12220

Assefa, T. T., Meuwissen, M. P. M. y Lansink, Oude A.G.J.M. (2015) "Price volatility transmission in food supply chains: A literature review”, Agribusiness: An International Journal 31: 3-13. DOI: 10.1002/agr.21380

Assefa, T.T., Meuwissen, M.P.M y Oude Lansink, A.G.J.M. (2017) "Price risk perceptions and management strategies in selected European food supply chains: An exploratory approach", Wageningen Journal of Life Sciences 80: 15-26. https://doi.org/10.1016/j.njas.2016.11.002

Beardsworth, A. y Bryman, A. (2004), "Meat consumption and meat avoidance among young people: an 11-year longitudinal study", British Food Journal 106 (4): 313-327. https://doi.org/10.1108/00070700410529573

Cáffaro Tommasiello, E.M., Rodríguez, M.G., Passucci, J., Purslow, P.P. y Latorre, M.E. (2021), "Producción, manejo, valoración, consumo y atributos de calidad de carne vacuna argentina: estudio mediante encuestas a carniceros", Ciencia \& Tecnología Agropecuaria 22(1): e1955. DOI: https://doi.org/10.21930/rcta.vol22_num1_art:1955

Capps, O. y R.M. Nayga (1991), "Demand for Fresh Beef Products in Supermarkets: A Trial with Scanner Data", Agribusiness 7(3): 241-251. 10.1002/1520-6297(199105)7:3<241::AID-AGR2720070306>3.0.CO;2-O

Charlebois. S. M. McCormick y M. Juhasz (2016), "Meat consumption and higher prices: Discrete determinants affecting meat reduction or avoidance amidst retail price volatility”, British Food Journal 118 (9): 2251 2270. DOI 10.1108/BFJ-03-2016-0121

Chavas, J. P. y A. Mehta (2004) "Price dynamics in a vertical sector", American Journal of Agricultural Economics 86: 1078-1093. . https://www.jstor.org/stable/4492793

Colella, F. y D.L. Ortega (2017), "Where's the beef? Retail channel choice and beef preferences in Argentina", Meat Science, doi: 10.1016/j.meatsci.2017.06.004

Eliçabe, H., Rodríguez, E.M. y Lupín, B. (2009), "Exportaciones de cortes de calidad de carne vacuna argentina. Un análisis de series de tiempo”, Ponencia presentada en la XL Reunión Anual de la Asociación Argentina de Economía Agraria, Universidad Nacional del Sur, 7-9 octubre, Bahía Blanca, Argentina

Fakari, B., Aliabadi, M.M.F., Mahmoudi, H., Kojori, M. (2016), "Volatility Spillover and Price Shocks in Iran's Meat Market",Custose @gronegócio on line 12 (2).

FAO, IFAD, IMF, OECD, UNCTAD, WFP, World Bank, WTO, IFPRI y UN HLTF (2011). Price Volatility In Food And Agricultural Markets: Policy Responses (Paris: OECD). Disponible en: http://www.oecd.org/dataoecd/ 40/34/48152638.pdf

Genchev, E., Y. Yarkova y D. Yarkov (2017), "Price determinants and interactions specifics in beef production in Bulgaria”, Agricultural Economics Review 18 (2): 97-113. http://www.eng.auth.gr/mattas/18_2_7.pdf

Hall, L., A. Schmitz y J. Cothern (1979), "Beef Wholesale-Retail Marketing Margins and Concentration", Economica 46 (183): 295-300. https://doi.org/10.2307/2553745

Kripfganz, S. y D.C. Schneider (2018). "ardl: Estimating autoregressive distributed lag and equilibrium correction models". Incluido en Proceedings of the 2018 London Stata Conference.

McFall Lamm, R. (1981), "Prices and Concentration in the Food Retailing Industry", The Journal of Industrial Economics 30 (1): 67-78. DOI: 10.2307/2098087 
Parcell, J.L. y T.C. Schroeder (2007), "Hedonic Retail Beef and Pork Product Prices”, Journal of Agricultural and Applied Economics 39 (1):29-46. DOI 10.22004/ag.econ.6622

Sahin, A., Yildirim, I. y Deniz, A. (2014), "A comparative study on urban and rural households' preferences of fresh mutton meat consumption (a case study in eastern region of Turkey-Heckman model)", British Food Journal 116 (12): 1897-1908. DOI: 10.1108/BFJ-06-2013-0161

Schroeder, T.C., T.L. Marsh, y J. Mintert (2000), Beef Demand Determinants, Tesis doctoral, Department of Agricultural Economics, Kansas State University.

Pace Guerrero, I., Berges, M. y Casellas, K. (2014), "Estimaciones de elasticidades de demanda para carnes y pescado en Argentina”, XLIX Reunión Anual de la Anales de la Asociación Argentina de Economía Política, 11-13 de Noviembre, Posadas, Argentina.

Piot-Lepetit, I. (2011) "Price volatility and price leadership in the EU beef and pork meat market", incluido en: I. Piot-Lepetit y R. M'Barek (eds.), Methods to Analyse Agricultural Commodity Price Volatility (New York: Springer Science and Business Media: 85-106).

Rabobank (2011), Rethinking The Food and Agribusiness Supply Chain; Impact of Agricultural Price Volatility on Sourcing Strategies (The Netherlands: Rabobank, 2011). Disponible en: http://hugin.info/133178/R/1549493/476482.pdf

Rossini, G. y Depetris Guiguet, E. (2008), “Transmisión vertical de precios en el sector de carne vacuna en Argentina", Revista de Análisis Económico 23(2): 3-119.

Serra, T. (2011) "Food scares and price volatility: The case of the BSE in Spain", Food Policy 36: 179-185. https://doi.org/10.1016/j.foodpol.2010.11.006

Surathkal, P. y C. Chung (2017): "Retail price responses to changes in wholesale prices in the US beef industry: differences among quality grades and primal cuts", Applied Economics 49(54): 5512-5522., DOI: 10.1080/00036846.2017.1311003

Uriarte, J.I., Ramírez Muñoz de Toro G.R., and Larrosa J.M.C. (2019), "Web scraping based online consumer price index: The IPC Online case”, Journal of Economic and Social Measurement 44(2-3): 141-159. DOI: 10.3233/JEM-190464

Van Asseldonk, M.A., Kuiper, W.E y Huirne, R.B. (2000) "Classical Swine Fever epidemic and price volatility", Proceedings of the 9th International Symposium on Veterinary Epidemiology and Economics (Breckenridge, Colorado).

Volpe, R., C. Risch y M. Boland (2017), "The Determinants of Price Adjustments in Retail Supermarkets", Managerial and Decision Economics 38 (1): 37-52. DOI: 10.1002/mde.2737

Wohlgenant, M.K. y J.D. Mullen (1987), "Modeling the Farm-Retail Price Spread for Beef”, Western Journal of Agricultural Economics 12(2): 119-125. https://www.jstor.org/stable/40987861 